\title{
Analysis of the spatial effects of inter-provincial air pollution in China
}

\author{
Zhang Yanyan ${ }^{1}$ \\ ${ }^{1}$ School of Economics, ShanDong women's university, 250300 Jinan, China
}

\begin{abstract}
Air pollution is a hot environmental issue that people have continued to pay attention to in recent years, and the government has spared no effort to strengthen its prevention. Haze is the result of the interaction between specific climatic conditions and human activities. Economic development and population agglomeration are important causes of smog on a large scale. Based on China's provincial haze pollution PM2.5 data from 2003 to 2017, Moran's I index and LISA scatter plot were used to analyze the spatial correlation of haze pollution. The results show that there is a positive spatial correlation between China's provincial haze pollution. Therefore, the problem of air pollution is a regional problem, and it is necessary for each region to strengthen regional joint prevention and control according to location conditions, natural conditions and economic conditions, and contribute to the prevention and control of smog.
\end{abstract}

\section{Introduction}

In recent years, haze weather has appeared more and more frequently throughout our country, especially in autumn and winter, which has seriously affected people's daily life. Air pollution will pose a serious threat to people's health and easily induce lung cancer, asthma, bronchitis and other diseases. Haze has a huge impact on the respiratory system, which has become the consensus of most people. In addition to the direct harm of haze pollution to the human body, it also has a negative impact on the economic growth and social development of an area. This is mainly reflected in an area developed by tourism. If the environmental quality is poor, it will seriously damage the image of the area and be detrimental to the economic development of the region. Haze pollution has become an urgent environmental problem due to its wide range and great harm. The prevention and control of haze is an urgent issue in current social development. However, only a comprehensive and in-depth understanding of the socio-economic root causes of air pollution can be implemented in a targeted manner.

After more than 30 years of development, China's economy has maintained sustained and rapid growth, and the process of industrialization has gradually advanced. However, behind the rapid economic development, the extensive development problems of "high investment, high consumption, and high pollution" are still prominent. This has led to frequent outbreaks of large-scale, high-intensity haze weather throughout China. In this context, we need to explore the spatial situation of haze pollution, and actively build a system and mechanism for joint prevention and control of regional air pollution, so as to actively respond to the problem of haze pollution.

\section{Literature review}

\subsection{Research on haze pollution from non-spatial perspective}

Many scholars have done research in this area. Ru and Lei (2014) analysed the causes and countermeasures of urban haze weather, and found that high energy consumption, low energy efficiency and export-oriented extensive economic development are important causes of haze pollution and transform economic growth. The way is the only choice to control the urban haze weather. Wang and $\mathrm{Xu}$ (2015) analysed the decoupling effect between environmental regulations and haze pollution. The study found that the intensity of environmental pollution supervision and environmental administrative control have a positive impact on the haze decoupling effect of corporate investment preferences. The decoupling effect of investment preferences has a negative impact. Guo Feng and Ren Yi (2018) used a panel threshold model to explore the threshold effect of economic growth on haze pollution. The study found that there is a significant non-linear relationship between economic growth and China's haze pollution. Under the threshold variable, as the economy continues to grow, China's smog pollution will increase.

\subsection{Research on Haze Pollution Based on Spatial Perspective}

Anselin, an internationally recognized expert in spatial measurement, specifically discussed the importance of

\footnotetext{
*Corresponding author:30055@sdwu.edu.cn
} 
spatial factors to environmental economic issues. Poon et al. (2006) used spatial measurement to study the impact of energy, transportation, and foreign trade on China's atmospheric environment, mainly focusing on smoke and dust, and confirmed that spillover effects do exist among Chinese provinces. Many early documents in China have conducted relevant research on the relationship between environment and economy. The topics involved include sustainable development, low-carbon economy, and research on the coordinated and sustainable development of regional economy and environment. As the problem of haze pollution has gradually become prominent In recent years, domestic scholars have paid more attention to the study of haze pollution. Shao Shuai et al. (2016) investigated the spatial correlation of smog pollution in my country and used dynamic spatial measurement methods to empirically identify the main factors of smog pollution. The spatial spillover effect of smog pollution in my country is obvious. The increase in the proportion of secondary industries, the increase in the proportion of coal consumption and the increase in population density will all increase the level of smog pollution.

\section{Research Methods}

\subsection{Global Moran's I index}

Goodchild (1992) pointed out that almost all spatial data have spatial dependence or spatial autocorrelation characteristics. In fact, the level of $\mathrm{PM}_{2.5}$ concentrations in a province is not only related to the level of smog emission in its "neighboring" areas, but may also be related to economic and social factors in other regions. The Global Moran Index is widely used to detect whether something is spatially related. Therefore, it was used to measure the spatial correlation between FDI and $\mathrm{PM}_{2.5}$ concentrations. Its formula is given as:

Moran'sI $=\frac{n \sum_{i=1}^{n} \sum_{j=1}^{n} w_{i j}\left(x_{i}-\bar{x}\right)\left(x_{j}-\bar{x}\right)}{\sum_{i=1}^{n} \sum_{j=1}^{n} w_{i j} \sum_{i=1}^{n}\left(x_{i}-\bar{x}\right)^{2}}=$
$\frac{\sum_{i=1}^{n} \sum_{j=1}^{n} w_{i j}\left(x_{i}-\bar{x}\right)\left(x_{j}-\bar{x}\right)}{S^{2} \sum_{i=1}^{n} \sum_{j=1}^{n} w_{i j}}$

Where $S^{2}=\frac{1}{n} \sum_{i=1}^{n}\left(x_{i}-\bar{x}\right)^{2}, \quad \bar{x}=\frac{1}{n} \sum_{i=1}^{n} x_{i}, \quad w_{i j}$ is the spatial weight matrix, $x_{i}, x_{j}$ are the observation values of $\mathrm{i}$ and $\mathrm{j}$ provinces and cities respectively. Moran's I $>0$ indicates a positive spatial correlation. Moran's I index is a rational number, and its value is between [-1-1], the larger the value, the more obvious the spatial correlation. Moran's $\mathrm{I}<0$ indicates negative spatial correlation, the smaller the value, the greater the spatial difference, otherwise, Moran's I $=0$, the space is random.

\subsection{Local Moran's I index}

In order to further analyze the local distribution of $\mathrm{PM}_{2.5}$ concentrations and FDI, this paper uses the local Moran's I to measure whether there is agglomeration effect among various provinces and cities. The formula for calculating the local Moran's I index is as follows:

$$
I_{i}=\frac{\left(x_{i}-\bar{x}\right)}{S^{2}} \sum_{i=1}^{n} w_{i j}\left(x_{i}-\bar{x}\right)
$$

Where $I_{i}$ is the local Moran's I index of province i, which measures the degree of correlation between province $\mathrm{i}$ and neighboring provinces' $\mathrm{PM}_{2.5}$ concentrations. This paper draws Moran scatter plots of $\mathrm{PM}_{2.5}$ concentrations in 2003 and 2017 (Fig.5 and Fig.6). The horizontal axis is the value of $\mathrm{PM}_{2.5}$ concentrations and the vertical axis represents is the spatial lag value of $\mathrm{PM}_{2.5}$ concentrations of each region. The provinces in the first and third quadrants indicate high-value gathering (high-high association) and low-value gathering (low-low association) respectively, which have strong spatial dependence. The second and fourth quadrants represent that the observation objects are negatively correlated, and there is no clustering phenomenon.

\subsection{Setting of spatial weight matrix}

Haze pollution is the result of a combination of many factors. However, climatic conditions are an important factor in the production of haze pollution. Haze can affect the haze of a certain place to neighboring areas through atmospheric circulation or wind. In this analysis Next, this paper mainly adopts the geographical distance spatial weight matrix to measure the spatial effect of haze pollution. The formula is as (4):

$w_{i j}= \begin{cases}\frac{1}{d_{i j}} & i \neq j \\ 0 & i=j\end{cases}$

Where $w_{i j}$ is the spatial weight matrix of geographic distance, $d_{i j}$ is the distance between province $i$ and province $j$, calculated from the latitude and longitude coordinates of the provincial capital city.

\section{Results}

\subsection{0verall measurement of haze pollution}

To further analyze the distribution of PM2.5 concentration in various provinces and cities, this paper uses ArcGIS10.2 to draw a quintile map of the spatial distribution of the mean $\mathrm{PM}_{2.5}$ concentration in each province in 2003, 2008, 2012, and 2017.

In Fig. 1-4, $\mathrm{PM}_{2.5}$ concentration has the characteristics of regional agglomeration in space. The concentration of PM2.5 in Beijing-Tianjin-Hebei-Shandong-Henan and surrounding areas has been higher than that in other areas, showing high agglomeration areas. These areas are dominated by second industry, with coal as the main energy source. As a result, the coal consumption per unit area is 4 times the national average. The pollutant emissions far exceeding the environmental carrying capacity are the main reason for the high $\mathrm{PM}_{2.5}$ concentration. What's more, 
semi-enclosed terrain conditions and adverse weather conditions are prone to accumulative pollution.

Although the government has been controlling environmental pollution, the haze pollution has not been effectively alleviated. Table 1 shows that the average $\mathrm{PM}_{2.5}$ concentration of haze pollution from 2003 to 2017 has been hovering around $40 \mu \mathrm{g} / \mathrm{m} 3$, and the highest value appeared in 2007. The maximum $\mathrm{PM}_{2.5}$ concentration was $84.3 \mu \mathrm{g} / \mathrm{m} 3$, which appeared in Tianjin in 2006 , followed by $82.2 \mu \mathrm{g} / \mathrm{m} 3$ in 2013 , which was also in Tianjin.

Table 1 Evolution of PM2.5 concentrations at the provincial level in China

\begin{tabular}{|l|l|l|l|l|}
\hline Year & Mean & Max & Min & Std. Dev \\
\hline 2003 & 36.58 & 61.30 & 11.10 & 14.05 \\
\hline 2004 & 36.50 & 57.60 & 12.60 & 12.83 \\
\hline 2005 & 39.61 & 64.40 & 14.00 & 14.28 \\
\hline 2006 & 43.79 & 84.30 & 13.50 & 18.01 \\
\hline 2007 & 44.41 & 78.90 & 14.60 & 17.71 \\
\hline 2008 & 42.65 & 74.50 & 15.30 & 15.47 \\
\hline 2009 & 42.27 & 78.00 & 13.40 & 15.77 \\
\hline 2010 & 42.02 & 72.4 & 12.1 & 16.28 \\
\hline 2011 & 39.83 & 71.10 & 14.70 & 15.40 \\
\hline 2012 & 37.28 & 62.30 & 10.2 & 14.07 \\
\hline 2013 & 43.09 & 82.20 & 15.00 & 17.46 \\
\hline 2014 & 43.42 & 76.90 & 13.10 & 16.68 \\
\hline 2015 & 40.32 & 69.80 & 13.10 & 15.67 \\
\hline 2016 & 36.16 & 67.30 & 12.90 & 14.37 \\
\hline 2017 & 42.21 & 68.40 & 16.70 & 13.93 \\
\hline
\end{tabular}

From the perspective of the spatial change of $\mathrm{PM}_{2.5}$ concentration (Fig.1), the range of provinces with heavy haze pollution expanded and increased from 2003 to 2008. The number of provinces with $\mathrm{PM}_{2.5}$ concentrations greater than $51.4 \mathrm{~g} / \mathrm{m} 3$ rose from 3 to 12 .

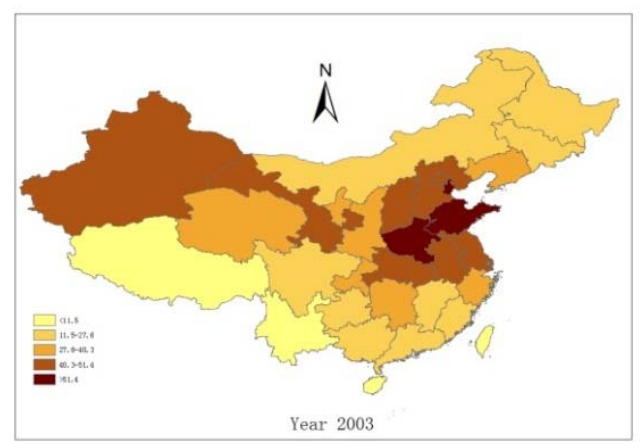

Fig.1 Distribution map of $\mathrm{PM}_{2.5}$ concentration in2008

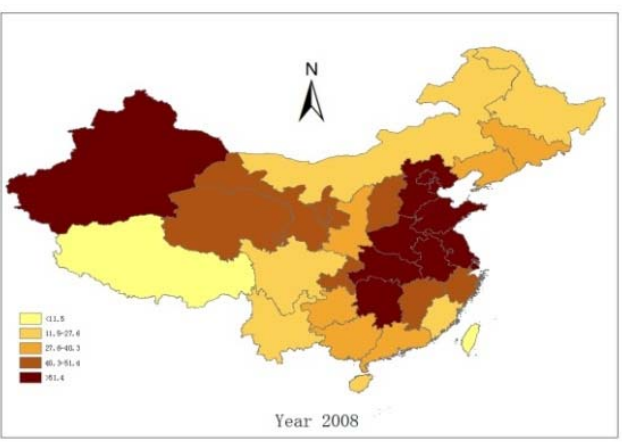

Fig.2 Distribution map of $\mathrm{PM}_{2.5}$ concentration in2008

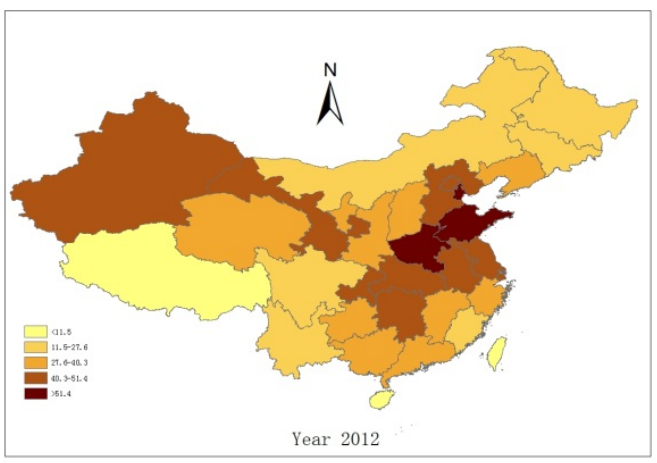

Fig.3 Distribution map of $\mathrm{PM}_{2.5}$ concentration in2012

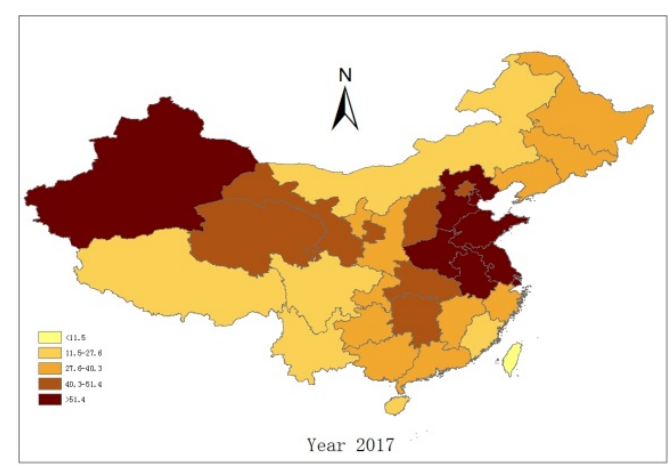

Fig.4 Distribution map of $\mathrm{PM}_{2.5}$ concentration in2017

\subsection{Spatial autocorrelation analysis of PM2.5}

We use the geographical distance spatial weight matrix (see formula 2) to measure the Moran's I index of $\mathrm{PM}_{2.5}$ concentrations in 30 provinces and cities.

Table 2. Moran's I index of PM2.5 concentrations from 2003 to 2017

\begin{tabular}{|c|c|c|}
\hline Year & Moran's I & Z Value \\
\hline 2003 & $0.188^{* * *}$ & 5.065 \\
\hline 2004 & $0.221^{* * *}$ & 3.543 \\
\hline 2005 & $0.246^{* * *}$ & 4.105 \\
\hline 2006 & $0.275^{* * *}$ & 4.769 \\
\hline 2007 & $0.274^{* * *}$ & 4.751 \\
\hline 2008 & $0.360^{* * *}$ & 4.441 \\
\hline
\end{tabular}




\begin{tabular}{|l|l|l|}
\hline 2009 & $0.257^{* * *}$ & 4.360 \\
\hline 2010 & $0.248^{* * *}$ & 4.153 \\
\hline 2011 & $0.374^{* * *}$ & 4.755 \\
\hline 2012 & $0.246^{* * *}$ & 4.099 \\
\hline 2013 & $0.285^{* * *}$ & 5.006 \\
\hline 2014 & $0.256^{* * *}$ & 4.341 \\
\hline 2015 & $0.298^{* * *}$ & 5.284 \\
\hline 2016 & $0.296^{* * *}$ & 5.262 \\
\hline 2017 & $0.268^{* * *}$ & 4.615 \\
\hline
\end{tabular}

As shown in Table 2, The Moran's I of PM2.5 concentrations from 2003 to 2017 were all positive and passed the $1 \%$ significance level test. This shows that the haze pollutant has a significant positive spatial correlation across the country. The PM2.5 concentration between provinces is interdependent.

As shown in Fig.5, in 2003, there were 14 provinces in the first quadrant ( $\mathrm{H}-\mathrm{H}$ association), and 10 provinces in the third quadrant (L-L association), three provinces lie in the second and fourth quadrants. As shown in Fig.6, in 2017, the provinces in each quadrant changed little. In general, the spatial association of PM2.5 concentrations in China is relatively significant, Beijing, Tianjin, Hebei, Shandong, Anhui, Henan, Hubei, Jiangsu, Shanghai, Gansu always locate in the first quadrant and Liaoning, Sichuan, Guangdong, Guangxi, Guizhou, Yunnan, Hainan, Heilongjiang, Jilin show low-value gathering, which can account for two-thirds of all provinces.

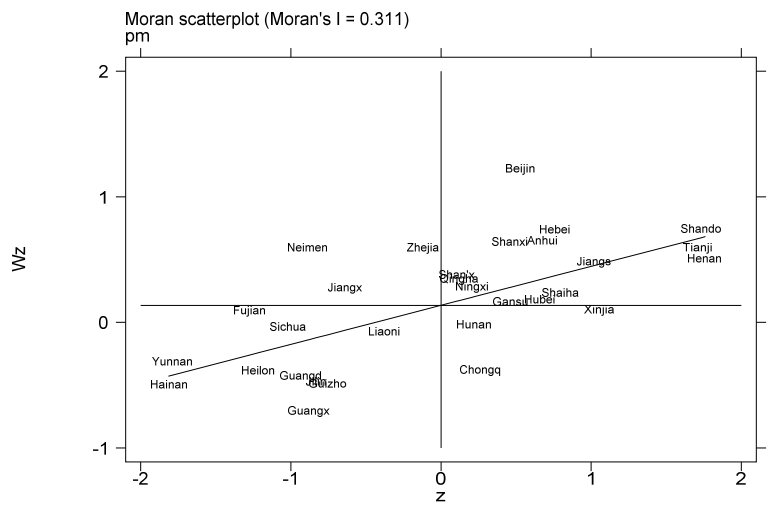

Fig.5. Local scatter plot of $\mathrm{PM}_{2.5}$ concentrations (2003)

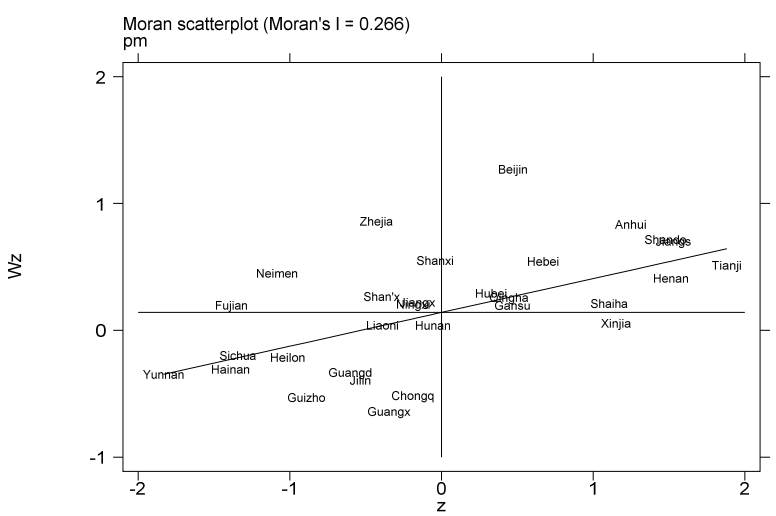

Fig.6. Local scatter plot of $\mathrm{PM}_{2.5}$ concentrations (2017)

\section{Conclusion}

Air quality has always been a hotspot for residents, seeking the source of haze pollution is an important prerequisite for rational haze control. This paper mainly selects panel data from 30 provinces in my country from 2003 to 2017. First, through the Moran index value and the Moran scatter plot, under the geographical weight matrix, it is verified that haze pollution has a significant positive spatial spillover effect. Due to the spatial effect of haze, we propose the following specific suggestions to prevent air pollution. First, the control of haze pollution should be based on the overall situation, based on the characteristics of different provinces, combined with the degree of economic development of the region and the degree of haze pollution, and differentiated implementation of haze control policies. According to the research conclusions of this article, haze pollution has significant space spillover effects. Faced with the regional, complex, and fluid characteristics of haze pollution, no province can stand alone. It must be achieved through regional air improvement to achieve real improvements in the spatial quality of the region. Second, Haze pollution has significant public and external characteristics. From this perspective, it is necessary to actively establish a cost sharing and benefit coordination mechanism between provinces, increase the willingness to cooperate, and implement effective fiscal transfer payments and sharing compensation.

\section{References}

1. Malinauskaite, D. Cook, B. Davíðsdóttir, H. Ögmundardóttir, and J. Roman, Ocean Coast. Manag., vol. 183,(2019)

2. Li, Y. Qi, C. Li, and X. Liu, J. Clean. Prod., vol. 209, pp. 1195-1205,( 2019)

3. He, M. Fan, and M. Zhou, J. Environ. Econ. Manage., vol. 79, pp. 18-39, (2016)

4. Burtraw, A. Krupnick, K. Palmer, A. Paul, M. Toman, and C. Bloyd, J. Environ. Econ. Manage., vol. 45 , no. 3 , pp. $650-673,(2003)$

5. B. Mao, C. Ao, J. Wang, B. Sun, and L. Xu, J. Clean. Prod., vol. 254, p. 120052, (2020)

6. G. Qiu, R. Song, and S. He, Sci. Total Environ., vol. 651, pp. 1114-1125, (2019)

7. L. Bai, L. Jiang, D. yang Yang, and Y. bin Liu, $J$. Clean. Prod., vol. 232, pp. 692-704, (2019)

8. Tang and Y. Zhang, J. Environ. Plan. Manag., vol. 59, no. 8, pp. 1473-1494, (2016)

9. M. C. Mirabelli, S. Ebelt, and S. A. Damon, Environ. Res., vol. 183, no. January, p. 109185, (2020)

10. Seung-Hoon Yoo et al. J. Environ. Plan. Manag., vol.86, pp.308-318 